\title{
Pan Bouyoucas : une écriture plurielle
}

Kathleen Kellett, Université Ryerson

Kirsty Bell, Université Mount Allison

L'œuvre de Pan Bouyoucas est indéniablement définie par la pluralité et la diversité. Diversité culturelle d'abord, puisque l'auteur lui-même est né au Liban et habite au Canada depuis 1963, et que certains de ses récits évoquent les origines grecques de ses parents. Diversité linguistique ensuite, puisque Bouyoucas est à la fois traducteur et romancier qui publie tantôt en français, tantôt en anglais. Pluralité littéraire enfin, puisque l'œuvre de Bouyoucas compte des romans, des récits, des nouvelles et des pièces de théâtre, et que l'auteur explore de nombreux sous-genres, du réalisme magique au roman policier. Depuis sa première publication, Le dernier souffle (1975) à sa plus récente Ce matin sur le toit de l'arc-en-ciel (2017), sa production se prête à des analyses portant sur des sujets d'une grande pertinence contemporaine : l'interculturel, la critique sociopolitique, les enjeux de la langue et de la traduction, le jeu avec les genres littéraires, la dynamique familiale, le réalisme magique et la question des origines, entre autres. L'œuvre de Bouyoucas voyage entre le Québec et la Grèce mais porte toujours sur le défi de sonder les enjeux $\mathrm{du}$ monde contemporain. Cependant, il existe peu d'articles critiques offrant des analyses approfondies des ouvrages de Bouyoucas, ou le situant dans le paysage littéraire canadien actuel.

La genèse de ce projet était la rencontre des co-éditrices au colloque de l'APLAQA (Association des professeur.e.s des littératures acadienne et québécoise de l'Atlantique) à Halifax en octobre 2014 où nous avons toutes les deux fait des communications sur Pan Bouyoucas. Suite à plusieurs discussions, nous avons décidé qu'un numéro spécial sur cet écrivain prolifique et divers, mais injustement méconnu, s'imposait. La directrice de Voix plurielles, Catherine Parayre, a gentiment accepté notre proposition de numéro et nous avons par conséquent le plaisir de présenter ici quatre articles soulignant la nature polyvalente de la production littéraire de Bouyoucas, précédés d'un entretien avec l'auteur, mené par Kathleen Kellett, avec la collaboration de Kirsty Bell.

L'entretien, «Pan Bouyoucas : l'écrivain, l'apprentissage, l'espace de l'écriture », nous donne un aperçu de son œuvre dans son entièreté ainsi que les « appartenances multiples » qui nourrissent sa vision du monde. Plus encore, l'autoportrait de l'auteur révèle sa croyance à la magie 
de la création littéraire, son amour des îles, sa connaissance intime de la ville cosmopolite de Montréal, son opposition passionnée au fanatisme et à l'intégrisme. Il nous présente un art poétique axé sur la précision et le dépouillement. Ses commentaires à propos de la genèse de ses propres écrits et des thèmes qui l'inspirent devront enrichir notre appréciation de son œuvre.

L'article de Kirsty Bell tient compte des interactions entre l'art visuel et les expressions de foi et de désir chez les protagonistes d'Anna pourquoi (2004). Alors que l'art visuel constitue un sujet de prédilection pour de nombreux romanciers contemporains, ce n'est pas typiquement le cas pour l'œuvre de Bouyoucas. Anna pourquoi se distingue donc des autres récits de Bouyoucas dans la mesure où ce roman est saturé de renvois au visuel : il y est question de cinéma, d'ex-voto, d'icônes et de graffiti. Ce recours à l'art dans Anna pourquoi n'est pas étonnant pour un écrivain qui a également une formation en art et en architecture, ce dont il parle dans l'entretien publié dans ce même numéro. L'article de Bell examine les manières dont l'image (et des avatars de l'image) élucide les pensées, la foi et les relations entre les personnages principaux du roman, une nonne sceptique, une novice zélée et un diacre peintre d'icônes.

L'article de Maria Geist explore l'altérité dans Le mauvais æeil (2015), notamment dans le cadre d'une petite île grecque où se déroule la trame narrative du roman. La culture de superstition dans cette île et la croyance au mauvais œil basculent dans une méfiance de l'Autre, voire dans la xénophobie. Geist s'intéresse aux manières dont Bouyoucas exploite l'ironie, les stéréotypes et les aptonymes afin d'examiner les questions de migration et d'immigration, et afin de remettre en cause le traitement de l'Autre comme bouc-émissaire. Il s'agit là de sujets importants et récurrents dans l'œuvre de Bouyoucas, et l'article de Geist constitue une analyse du Mauvais œeil comme une sorte d'étude de cas.

Pour sa part, Kathleen Kellett s'intéresse à la polyvalence des genres littéraires chez Bouyoucas en analysant les motifs du roman policier dans La vengeance d'un père (1997) et Cocorico (2011), l'un situé à Montréal et l'autre sur l'île de Nysa en Grèce, mais tous les deux axés sur l'opposition entre l'abject, au sens kristevien, et l'intègre, représenté par l'idéal de rationalité et d'altruisme incarné par le personnage du détective. Par contre, cette intégrité idéale doit se défendre contre l'abject qui est entraîné, d'une part, dans La vengeance d'un père par des conflits d'ethnie et de classe sociale dans un Montréal post-référendaire et, d'autre part, dans Cocorico, par l'inexorable chute dans la démence sénile. 
Pour clore ce numéro, Simon Harel part de l'analyse du récit L'Autre (2001), finaliste au Prix du Gouverneur-général en 2001, pour situer 1'œuvre de Bouyoucas dans son contexte québécois et mondial. Il souligne les rapports entre ces écrits et ceux d'Emile Ollivier, de Wajdi Mouawad, d'Antonio d'Alfonso et de N.S. Naipaul en tant qu'écriture migrante. À partir de sa théorie des braconnages identitaires, Harel offre une analyse de L'Autre de Bouyoucas, récit où l'Autre intervient dans la vie de soi, afin d'usurper sa place. Pour Harel, loin d'être tragique ou traumatique, chez Bouyoucas, la dépossession de l'exil donne lieu à une exploration ludique de la nature transculturelle et transgénérationnelle de la migration. Le rapport de l'être humain à l'oïkos est au centre du questionnement identitaire.

En guise de conclusion, nous tenons à remercier tous nos collègues, surtout l'éditrice de Voix plurielles, Catherine Parayre, de leur patiente collaboration. Ceux qui ont eu la gentillesse d'évaluer les articles méritent une mention spéciale car leurs commentaires ont été fort précieux pour la révision des textes. Enfin, nous remercions Pan Bouyoucas de sa générosité en nous permettant de l'interviewer au mois de mars 2016 à Montréal, ainsi que d'avoir revu et retravaillé le texte par la suite. Nous espérons que de futurs chercheurs s'inspireront de ce numéro pour offrir des études approfondies et diverses de cet auteur québécois. 